\title{
Von Verfolgung hin zu Betreuung: Bedeutungswandel schweizerischen Heroinkonsums in zeit- und lebensgeschichtlicher Perspektive
}

Hans-Peter von Aarburg

Michael Stauffacher

\author{
Changed Benefits and Harm of Heroin Use in Switzerland through a Policy Shift \\ from Repression to Care
}

\section{Zusammenfassung}

Wir untersuchten in der Schweiz vorkommende Konsummuster von Heroin in ihrem Wandel während der letzten drei Jahrzehnte. Dabei fragten wir vor allem nach dem Einfluss einer hierzulande inzwischen mehr von Hilfe als von Repression geprägten Heroinpolitik. Wir verwendeten historische Dokumente, daneben aber auch sekundärstatistische Daten aus dem sozialen und polizeilichen Bereich und vor allem biografische Interviews mit rund 50 Personen. Wir fokussieren an der Schnittstelle von Zeitund Lebensgeschichte vor allem Nutzen und Schaden spezifischer Konsummuster für Einzelne und die Gesellschaft. Den Bedeutungswandel des Heroinkonsums teilten wir in folgende sozialgeschichtliche Perioden: „Counter culture“ (1965 - 1975) „No future“ (1975 - 1985) - „Public hell“ (1985-1995) - „Controlled disease“ (ab 1995).

\section{Schliuisselwörter}

Heroin · Nutzen · Schaden · Geschichte · Schweiz

\section{Abstract}

We assessed historically changing patterns, meanings and demographic aspects of heroin use in Switzerland during the last three decades. We investigated in particular the influence of the Swiss drug policy on the attractiveness of such changed forms of heroin use. Three complementary types of data have been combined in the present study: documents from available literature and archives, longitudinal statistical data from repression and care agencies and general population surveys, and biographical interviews with around 50 current or former heroin users. We distinguish four time periods of heroin use in Switzerland: "Counter culture" (1965 - 1975) - "No future" (1975-1985) "Public hell" (1985-1995) - "Controlled disease" (1995-present). By scrutinizing individual and societal harm and benefits of particular patterns of heroin use, we found many unexpected unconscious mechanisms of behaviour. On the whole, caring has - to a large extent - sabotaged the rebellious image of heroin use by transforming it into a disenfranchising chronic disease. Heroin now serves much less as a frightening scapegoat for society.

Key words

Heroin $\cdot$ benefit $\cdot$ harm $\cdot$ history $\cdot$ Switzerland

Danksagung

Dieser Artikel basiert auf einem vom Schweizerischen Bundesamt für Gesundheit finanzierten Forschungsprojekt. Die Resultate wurden an der 14. Konferenz der ESSD (European Society for Social Research on Drugs) vom Oktober 2003 präsentiert. Wir danken den Teilnehmenden für ihre wertvollen Kommentare und Ergänzungen.

Institutsangaben

Ethnologisches Seminar, Universität Basel

Korrespondenzadresse

Hans-Peter von Aarburg • Michael Stauffacher· Ethnologisches Seminar, Universität Basel · Münsterplatz 19 . 4051 Basel·Schweiz·E-mail: hp.vonaarburg@unibas.ch 
Die Ausgangsfrage als eine methodologische

Herausforderung

Heroin als Gassendroge war noch um 1970 in der Schweiz weit gehend unbekannt, dann verbreitete sich sein Konsum während etwas mehr als zwei Jahrzehnten zeitweise sprunghaft [1-8]. Seit den frühen 90er-Jahren nun scheint sich die Zahl der Heroin Konsumierenden hierzulande insgesamt stabilisiert zu haben, die Zahl der neu Einsteigenden geht seither deutlich zurück [9-15]. Neben diesen zahlenmäßigen Veränderungen haben sich in den letzten drei Jahrzehnten vor allem auch die typischen Konsummuster unablässig gewandelt [3, 16-20]. Dabei haben sich neben den Drogenkombinationen und Anwendungsformen auch Umstände und Bedeutungen des Konsums viel stärker verändert, als das selbst direkt Betroffene meist wahrnehmen. In der Wahrnehmung von Fachleuten ebenso wie in der Darstellung der Medien gilt Heroin in der Schweiz inzwischen als „Loser-Droge“, die heutige Jugendliche vergleichsweise wenig fasziniert.

Wir untersuchten, wie sich das Image des Heroinkonsums hierzulande während der letzten drei Jahrzehnte verändert hat. Dabei rekonstruierten wir Bedeutungen in der Perspektive der Heroin Konsumierenden selbst und der sie umgebenden Gesellschaft. An den stark gewandelten Konsummustern interessierte uns vor allem, welche spezifischen Formen von Nutzen und Schaden sie bedeuteten. Wir versuchten hier, subjektiven, kommunikativ vermittelten Sinn zu rekonstruieren, wie er dem Konsum bestimmter pharmakologischer Substanzen in spezifischen Einnahmearten und sozialen Kontexten lebensgeschichtlich zukommt. Anders formuliert, fragten wir nach der jeweiligen Attraktivität spezifischer Formen des Heroinkonsums, fragten danach, welche subjektiven Bedeutungen diese für lebensgeschichtliche Identitätskonstruktionen haben. Nutzen und Schaden erweisen sich dabei als oft höchst relative Kategorien. So kann ein gesundheitlich und sozial offensichtlich selbstschädigendes Verhalten durchaus auch einen subjektiven Nutzen für die persönliche Identität bedeuten. In Einzelfallanalysen suchten wir neben offensichtlichen auch nach versteckten, neben bewussten auch nach unbewussten Formen des Nutzens und Schadens.

Psychiatrische Herangehensweisen beschränken sich meist auf solche individual- und sozialpsychologische Betrachtungsweisen. Wir untersuchten hier aber auch Entwicklungen in der zeitgeschichtlichen Dimension, mit denen sich einzelne Individuen lebensgeschichtlich auseinander zu setzen haben. Es sind damit allgemeine wirtschaftliche, staatliche und kulturelle Entwicklungen gemeint, hier vor allem aber auch eine stark veränderte Drogenpolitik. Neben einer handlungstheoretischen Perspektive nahmen wir also auch eine soziostrukturelle Perspektive ein und stellten dabei eine Entwicklung von einem verfolgten hin zu einem betreuten Heroinkonsum fest. Im Folgenden werden hier gleich anschließend zuerst zeitgeschichtliche Perioden dieses Entwicklungsprozesses beschrieben. Wir versuchten dann Nutzen und Schaden spezifischer Konsumformen auch aus einer gesellschaftlichen Perspektive zu rekonstruieren. Methodologisch berufen wir uns dabei auf die Ethnopsychoanalyse, welche unbewusste Mechanismen auch auf einer soziostrukturellen Ebene untersucht $[22,23]$.
Wiederum in einer individuellen Perspektive fragten wir, wie Konsumierende hierzulande mit dem allmählichen Wandel von einem vorherrschend verfolgten hin zu einem vorherrschend betreuten Heroinkonsum umgehen.

Alle hier gewonnenen Erkenntnisse haben zwar grundsätzlich eher den Charakter von Indikatoren als von schlüssigen Beweisen, in ihrer Dichte und durch die „Triangulation“ von Daten ganz unterschiedlicher Art führen sie aber doch zu empirisch recht gefestigten Aussagen.

\section{Daten und ihre Interpretation}

Um empirische Daten zu gewinnen, verwendeten wir drei methodisch sich ergänzende Zugänge: Informationen zum allgemein zeitgeschichtlichen und drogenpolitischen Hintergrund erarbeiteten wir mit Literatur- und Dokumentenrecherchen. Beobachtungen und Fakten aus vielen Dimensionen bleiben hier allerdings unerwähnt, denn die Gesamtheit aller möglicher Einflussfaktoren ist nicht zu bewältigen. Einen zweiten Zugang bildeten sekundärstatistische Analysen. Anhand unterschiedlichster Statistiken erfasste Michael Stauffacher quantitativ messbare Entwicklungen: einerseits die Angebotsentwicklung der Drogenhilfe, anderseits epidemiologische Aussagen zum Konsumbeginn zu verschiedenen Zeitpunkten, wie auch die Verbreitung des Drogenkonsums (Inzidenz und Prävalenz). Herangezogen wurden Daten aus dem Behandlungssystem, von allgemeinen Bevölkerungsbefragungen wie auch aus dem Repressionsbereich. Den dritten Zugang der Studie bildeten biografische Interviews. Hans-Peter von Aarburg hatte bereits Mitte der 90er-Jahre in Zürich eine ethnografische Untersuchung zum damals neu aufgekommenen Folienrauchen gemacht [16] und konnte viele der damals interviewten Jugendlichen erneut für Interviews gewinnen. Er hatte sie einst meist ohne institutionelle Vermittlung kennen gelernt. Neben privaten Kontakten fand er nun vor allem über Einrichtungen des Drogenhilfebereichs (Kontakt- und Anlaufstellen, Heroinabgabeprogramme, stationäre Therapieeinrichtungen) Personen, die ihren Heroinkonsum zu frühen, mittleren und späten Zeitpunkten der hier interessierenden Periode begonnen, inzwischen abgebrochen oder mit unterschiedlichen Konsummustern fortgeführt haben. Interviewpartnerinnen und -partner wurden im Sinne des „Theoretical Sampling“ ausgewählt, nicht nach Kriterien statistischer Repräsentation [24]. Es entstand ein Corpus von insgesamt 120 Interviews mit etwa 50 unterschiedlich intensiv befragten Personen. Ein großer Teil der befragten Personen nahm ihren Konsum um 1990 herum auf. Unter den Interviewten befinden sich deutlich mehr Männer als Frauen. Alle Interviews mit einer Gesamtdauer von rund 140 Stunden wurden auf Tonband aufgenommen und wörtlich transkribiert.

Konsummuster können einigermaßen zuverlässig erfasst werden. Viel schwieriger ist es aber, deren Bedeutungen auf einer individuellen und gesellschaftlichen Ebene zu rekonstruieren. Handelnde Individuen wissen ja oft selbst nicht recht, warum sie etwas tun und was es ihnen letztlich bedeutet. Das gilt ganz besonders für einen in sich so widersprüchlichen Bereich, wie ihn der selbstschädigende Drogenkonsum darstellt. So erweisen sich geäußerte Handlungsmotive bei näherer Betrachtung oft als 
rationalisierende Selbst- und Fremdtäuschungen, die stark von aktuellen Lebenssituationen und auch von kommunikativen Umständen abhängen. Tatsächliche Motive müssen deshalb oft aufgrund von Indizien erschlossen werden, die in erzähltem oder beobachtetem Handeln fassbar werden. Dabei müssen Feststellungen zu Nutzen und Schaden spezifischer Konsummuster vor allem auch auf einer Ebene wenig oder gar nicht bewusster Motive erarbeitet werden. Dies gilt übrigens ebenso sehr für die individuelle wie für die gesellschaftliche Ebene.

In den hier nicht dargestellten Einzelfallanalysen wurden dann lebensgeschichtliche Bedeutungen des Heroinkonsums in ihren zeitgeschichtlichen Verschränkungen zu rekonstruieren versucht. Eine Buchpublikation wird einige dieser Einzelfälle aus unterschiedlichen Perioden detailliert darstellen.

\section{Zeitgeschichtliche Perioden}

Die vier zeitgeschichtlichen Perioden wurden vor allem aufgrund von Ereignissen und Entwicklungen in der Stadt Zürich gegeneinander abgegrenzt. Entsprechende Beobachtungen wären in abgeschwächter Form aber auch in anderen Schweizer Städten zu machen, zudem prägten die Zürcher Erfahrungen die Drogenpolitik auf Bundesebene entscheidend. Die Dichte der vier Zeitabschnitte wird hier dadurch vereinfacht dargestellt, dass Elemente wechselnder Konsummuster nur in drei wichtigen Dimensionen vergleichend aufgelistet werden.

\section{„Counter-culture“ (1965-1975)}

Substanzen: Wie in anderen Ländern waren auch in der Schweiz LSD und Cannabis die Leitdrogen der Protestgeneration jener Jahre. Bis zum „Ölschock“ von 1973 entsprach einer damals überbordenden Wachstumseuphorie spiegelbildlich eine oft fundamentale Zivilisationskritik. Heute oft vergessen wird, dass sowohl im gesellschaftlichen Mainstream wie auch in der Gegenkultur der Amphetaminkonsum damals stark verbreitet war. Als Gassendroge wurde Amphetamin auch in der Schweiz injiziert. Diese Anwendungsform war bereits eingeführt, als man in geringerem Maße auch Opiumtinkturen und Morphium aus Apothekeneinbrüchen intravenös zu konsumieren begann. Anfangs der 70er-Jahre wurden diese Opiate neben dem rasch vordringenden Heroin allerdings bald zu exotischen Raritäten.

Verfolgung: Alle damals fremdartigen Drogen wurden anfänglich wenig differenziert. Ihr Konsum wurde als extreme Gesundheitsgefahr und gleichzeitig auch als kulturelle Subversion geächtet und verfolgt.

Betreuung: Es entstanden die ersten therapeutischen Wohngemeinschaften, wobei sich oft engagierte junge Erwachsene aus einem „alternativen“ Umfeld um fast gleichaltrige Drogen Konsumierende kümmerten. Die noch junge Sozialpsychiatrie richtete erste Beratungsstellen ein.

\section{„No future“ (1975 - 1985)}

Substanzen: Heroin wurde ursprünglich meist von Konsumierenden in kleinen Mengen aus dem Ausland in die Schweiz gebracht und hier unter Kollegen weiterverkauft. Es handelte sich um südostasiatisches Heroinsalz, das injiziert wurde. Die Zahl der Kon- sumierenden stieg langsam, aber stetig. Methadon spielt in den Konsummustern noch kaum eine Rolle.

Verfolgung: Während der so genannten Zürcher Jugendunruhen wurde Heroin zu einem wichtigen Thema. Die „Szene“ wurde von verschiedenen öffentlichen Plätzen verdrängt und schließlich vorübergehend im heiß umstrittenen Autonomen Jugendzentrum (AJZ) geduldet, bis dieses abgerissen wurde. Autoritäre und liberale Interpretationen der jugendlichen Unrast rangen um die Definitionsmacht. Die polizeiliche Verfolgung der Konsumierenden nahm stark zu, begann aber an ihre Grenzen zu stoßen.

Betreuung: Das Behandlungsangebot bestand vor allem aus Entzugsstationen und abstinenzorientierten, stationären Langzeittherapien. Viele von diesen waren stark von gesellschaftskritischen, andere von religiösen Idealen geprägt. Polizeiliche und therapeutische Interventionen versuchten je auf ihre Weise, das Ziel drogenfreien Lebens durchzusetzen. Methadon wurde auch als „ambulante Maßnahme“ - zwar verordnet, die Indikation war aber noch eng und streng kontrolliert. Gleichzeitig entstand im AJZ vorübergehend der erste, von Freiwilligen betreute Injektionsraum der Schweiz [25, 26]. Das auch viele Schweizer Jugendliche faszinierende Buch und der entsprechende Film über die Berliner „Kinder vom Bahnhof Zoo“ erwähnten konsequent keine Drogenhilfeeinrichtung irgendwelcher Art [27].

\section{„Public hell“ (1985 - 1995)}

Substanzen: Afghanische Heroinbase - sie ist direkt rauchbar, aber nur nach Aufkochen mit Ascorbinsäure injizierbar - verdrängte allmählich das frühere Heroinsalz. Der Handel wurde zunehmend von ethnisch strukturierten Banden professionalisiert. Konsumierende führten nun meist nur noch untergeordnete Tätigkeiten im Drogendeal aus. Ende der 80er-Jahre begann sich auch das Kokain massiv zu verbreiten. Es wird seither in vielfältigen Varianten mit Heroin kombiniert, v. a. gleichzeitig oder einige Zeit vor diesem injiziert oder als als „Freebase“ bzw. als „Crack-Cocaine“ geraucht. In vielen Konsummustern verdrängte Kokain das Heroin als „Hauptdroge“ nachhaltig. Häufig wird seit dieser Zeit Heroin nun auch mit Rohypnol und anderen Medikamenten kombiniert. Methadon wird immer mehr zu einem festen Bestandteil in vielen Konsummustern. Kombinationen mit LSD und Ecstasy finden sich auch heute noch meist nur zu Beginn des Heroinkonsums. In der zweiten Hälfte der 80erJahre schnellten die Zahlen der Konsumierenden bei immer noch sehr hohen Preisen in die Höhe. Die Gassenpreise für Heroin begannen erst anfangs der 90er-Jahre von etwa 500 auf unter 100 Franken zu fallen, dies bei stark gestiegenem Reinheitsgrad $[16,28]$. Bereits kurz vor diesem Preiszerfall war neben dem Injizieren das „Folienrauchen“ aufgekommen, das vorübergehend auch sozial völlig unauffällige Jugendliche in seinen Bann zog.

Verfolgung: Ganz in der Nähe des abgerissenen AJZ entstand in den späten 80er-Jahren eine weltberüchtigt gewordene offene Drogenszene. Nach einem ersten polizeilichen Schließungsversuch verschob sich diese lediglich um einige hundert Meter; Kriminalität und Verelendung auch in einem weiteren Umfeld wuchsen dort für alle Beteiligten während gut zwei Jahren ins Unerträgliche. Die Situation war nicht mehr polizeilich zu lösen, 
das „Drogenproblem“ absorbierte die Schweizer Öffentlichkeit in einem inzwischen kaum mehr vorstellbaren Maß. Die zweite Schließung 1995 gelang, weil inzwischen soziale Hilfeeinrichtungen und Polizei die verfahrene Situation bzw. ihre je beschränkten Möglichkeiten erkannt und ihre Strategien miteinander zu koordinieren begonnen hatten.

Betreuung: Vorerst um die weitere Ausbreitung des HI-Virus zu stoppen, wurde seit Mitte der 80er-Jahre mit dem Aufbau einer Schaden mindernden sozialmedizinischen Infrastruktur für intravenös konsumierende Drogenabhängige begonnen. Viele ursprünglich private Initiativen sozialmedizinischer und sozialarbeiterischer Drogenhilfe wurden im Laufe der Jahre durch die öffentliche Hand übernommen und institutionell konsolidiert. Ziel war es, die gesundheitliche und soziale Verelendung intravenös Heroin Konsumierender zu verringern und gleichzeitig auch die öffentliche Ordnung wiederherzustellen. Folienrauchende waren lange Zeit wenig betreut. Schadenminderung und Überlebenshilfe wurden auch auf Bundesebene zu einem integrierten Pfeiler der offiziellen Drogenpolitik neben Prävention, Therapie und Repression [29]. Als Einzelmaßnahme hat wohl die Herabsetzung der Zugangsschwellen zur Methadonsubstitution die Konsummuster langfristig am tiefgreifendsten verändert.

\section{„Controlled disease“ (1995-2003)}

Substanz: Der Heroinpreis ist weiter zerfallen und liegt 2003 um 50 Franken pro Gassengramm, wobei in den letzten Jahren allerdings auch der Reinheitsgrad gesunken ist. Zu einem noch wichtigeren Element in individuellen Konsummustern ist Methadon geworden. Wohl eine Mehrzahl der Abhängigen setzt es inzwischen gezielt ein, um ihren Heroinkonsum zu kontrollieren und seine gesundheitlichen und sozialen Folgen einigermaßen im Griff zu behalten. Die Muster der miteinander konsumierten Substanzen werden noch individueller und vielfältiger, wichtig sind dabei vor allem Kokain, Medikamente und Alkohol. Eine weiterhin untergeordnete Rolle spielen LSD und Ecstasy. Die Gesamtzahl der Heroin Konsumierenden blieb in der Größenordnung stabil, die Zahl der Neueinsteigenden aber nimmt markant ab. Das durchschnittliche Alter der Heroin Konsumierenden steigt stetig an.

Verfolgung: Ein Hauptziel der polizeilichen Verfolgung besteht nun vor allem darin, Deal und Konsum des Heroins von öffentlichen Räumen zu verbannen und so die Öffentlichkeit vor Störungen und Belästigungen zu schützen. Weiterhin können zwar auch einfache Konsumierende angehalten und gebüßt werden, die Drogenfahndung fokussiert nun aber vermehrt Händlernetze. Richtete sich der Fokus einst auf jugendliche Konsumierende, zielt er nun eher auf ethnisch strukturierte Dealerbanden.

Betreuung: Sozialmedizinische Infrastruktur und sozialarbeiterische Stabilisierungs- und Rehabilitationsmaßnahmen wie begleitetes Wohnen und Beschäftigungsprogramme wurden vorerst weiter konsolidiert, in den letzten Jahren droht nun allerdings ein Abbau. Es werden vermehrt Invalidenrenten zugesprochen, wobei die psychiatrischen Begriffe der Komorbidität bzw. der Doppeldiagnose angewendet werden. Methadonbehandlungen sind zum Regelfall geworden. Die früher alleinigen und insgesamt wenig erfolgreichen Therapieformen abstinenzorientierter Ausrichtung werden nun nur noch nach umfassen- der Abklärung und guter Prognose von den Kostenträgern bezahlt. Stationäre Einrichtungen müssen ihr Angebot an die enger und individueller gewordene Nachfrage anpassen und geraten unter finanziellen Druck. Die bereits 1994 gestarteten Heroinprogramme werden fester, auch durch eine Volksabstimmung legitimierter Teil des therapeutischen Angebots. Die „heroingestützten Behandlungen“ erfreuen sich aber weniger großer Attraktivität unter Abhängigen, als von institutioneller Seite erwartet wurde. Das Drogenhilfesystem insgesamt bleibt auf den Heroinkonsum ausgerichtet, richtet sich nun aber vermehrt auch an Folienrauchende. Kokainkonsum wird nur beiläufig mitbetreut, insbesondere gibt es keine Substitutionsprogramme für Kokain. Die gelegentlich praktizierte ärztliche Verschreibung des Amphetamins Ritalin ${ }^{\circledR}$ als Ersatz für Kokain an polytoxikomane Drogenabhängige bewegt sich in einer Grauzone.

Nutzen und Schaden verfolgter und betreuter Konsummuster

Die im Folgenden tabellarisch zusammengestellten Aussagen sind holzschnittartig vereinfacht, vor allem reicht der Platz hier nicht, um sie durch Interviewdaten zu belegen (Tab.1). Es soll hier vor allem darauf hingewiesen werden, in welchen Dimensionen und in welche Richtungen wir Antworten auf die aufgeworfene Fragestellung gesucht haben.

Typologisch vereinfacht werden hier verfolgte und betreute Konsummuster einander gegenübergestellt. Auf einer zeitgeschichtlichen Achse hat sich der Heroinkonsum in der Schweiz eindeutig von einem Modus der Verfolgung hin zu einem Modus der Betreuung verändert. Beide Elemente sind auch heute noch vorhanden, ihre Gewichte haben sich aber verschoben. Unterschiedliche Konsumentengruppen dürften zudem entsprechend ihrer jeweiligen Ressourcen ungleich stark den Risiken polizeilicher Verfolgung oder möglicherweise entmündigender Betreuung ausgesetzt sein. Auch verändern sich die Anteile der beiden Bereiche im Laufe jeder Konsumkarriere ständig - etwa gibt es auch heute noch keinen betreuten Konsumbeginn.

Besonders lohnend dürfte es sein, schwer verständliche Konsummuster unter dem Aspekt der Selbstbestimmung zu betrachten, und nach dem entsprechenden Nutzen zu fragen. So etwa versuchen inzwischen viele Methadonbezieher und -bezieherinnen oft gegen ausdrücklichen ärztlichen Rat, ihre tägliche Dosis abzubauen. Von niedrigen Dosen abgeraten wird in vielen Fällen aufgrund der Beobachtung, dass es dabei leichter zu punktuellen Rückfällen und regelmäßigem Beikonsum kommt. Offensichtlich ziehen viele Konsumierende aber ein selbstverantwortetes Ringen mit den Tücken der Abhängigkeit einem problemlos hohen Zugedecktsein mit dem Opioid vor. Man kann sich auch fragen, ob die stark gestiegene Attraktivität des Kokains neben pharmakologischen Eigenschaften auch damit zu tun hat, dass diese Substanz noch weit gehend unbetreut ist. An der gegenwärtigen Attraktivität des Kokains irritiert übrigens auch, dass es hierzulande um 1990 erst etwa halb soviel wie Heroin kostete, inzwischen aber zu dessen dreifachem Preis gehandelt wird. Auf den ersten Blick völlig unverständlich erscheint auch, dass es Personen gibt, die in Behandlungsprogrammen reines Heroin kostenlos und in frei verhandelbaren Dosen bekommen und sich 
Tab. 1 Verfolgter und betreuter Heroinkonsum

\begin{tabular}{|c|c|c|c|}
\hline \multicolumn{4}{|c|}{ verfolgter Konsum } \\
\hline & & für Konsumierende & für Gesellschaft \\
\hline \multirow[t]{3}{*}{ Nutzen } & bewusst & Genuss & Gering \\
\hline & & $\begin{array}{l}\text { Analgetikum } \\
\text { Antidepressivum } \\
\text { rebellische Identität }\end{array}$ & $\begin{array}{l}\text { am Rand evtl. Impulse zur Reflexion vorherrschender } \\
\text { sozialer Werte }\end{array}$ \\
\hline & unbewusst & $\begin{array}{l}\text { heldenhafte, angstlustvolle Herausforderungen } \\
\text { sich als verfolgtes Opfer fühlen } \\
\text { Sucht als Halt und Entlastung von Freiheit }\end{array}$ & $\begin{array}{l}\text { Feindbild entlastet als Sündenbock von der Analyse } \\
\text { tiefer liegender gesellschaftlicher Probleme }\end{array}$ \\
\hline \multirow[t]{2}{*}{ Schaden } & bewusst & $\begin{array}{l}\text { hohe, aber selbstverantwortete Risiken } \\
\text { sozialer Ausschluss unter dem Etikett: „bad“ } \\
\text { Krankheit } \\
\text { Tod }\end{array}$ & $\begin{array}{l}\text { Bedrohung sozialer Werte und öffentlicher Ordnung } \\
\text { Konsumierende als Subversive gefürchtet } \\
\text { hohe finanzielle Kosten der Repression }\end{array}$ \\
\hline & unbewusst & $\begin{array}{l}\text { bedrohtes Selbstwertgefühl durch } \\
\text { selbstverschuldeten Autonomieverlust }\end{array}$ & $\begin{array}{l}\text { Blindheit für soz. Probleme, die durch } \\
\text { Drogenprobleme überblendet werden }\end{array}$ \\
\hline \multicolumn{4}{|c|}{ betreuter Konsum } \\
\hline & & für Konsumierende & für Gesellschaft \\
\hline \multirow[t]{2}{*}{ Nutzen } & bewusst & $\begin{array}{l}\text { reduzierter Genuss } \\
\text { Analgetikum } \\
\text { Antidepressivum } \\
\text { reduzierte Risiken }\end{array}$ & $\begin{array}{l}\text { öffentliche Ordnung wiederhergestellt } \\
\text { stolz auf liberal offene Wohlfahrtspolitik }\end{array}$ \\
\hline & unbewusst & $\begin{array}{l}\text { Sucht als Krankheit dispensiert von vielen } \\
\text { Pflichten } \\
\text { Sucht als Halt und Entlastung von Freiheit }\end{array}$ & $\begin{array}{l}\text { als psychiatr. Gesundheitsprobleme umdefinierte } \\
\text { soziale Probleme entlasten von deren tiefergehender } \\
\text { Analyse }\end{array}$ \\
\hline \multirow[t]{3}{*}{ Schaden } & bewusst & $\begin{array}{l}\text { reduzierte, institutionell abgefederte Risiken } \\
\text { sozialer Ausschluss unter dem Etikett „mad“ }\end{array}$ & $\begin{array}{l}\text { lästige Störung der Öffentlichkeit } \\
\text { abhängig Konsumierende }\end{array}$ \\
\hline & & $\begin{array}{l}\text { umfassende institutionelle Kontrolle entmündigt } \\
\text { und bedroht das Selbstwertgefühl }\end{array}$ & $\begin{array}{l}\text { erscheinen als lästige chronisch Kranke } \\
\text { hohe Wohlfahrtskosten }\end{array}$ \\
\hline & unbewusst & $\begin{array}{l}\text { unheldenhaftes Ringen um Restautonomie als } \\
\text { Abwehr disziplinierender Unterwerfung, von den } \\
\text { Institutionen oft als Unreife interpretiert }\end{array}$ & $\begin{array}{l}\text { Bedrohung der Grundwerte liberal offener Gesellschaf- } \\
\text { ten durch umfassende institutionelle Kontrolle indiv. } \\
\text { Freiheit }\end{array}$ \\
\hline
\end{tabular}

trotzdem dazu noch gelegentlich für teures Geld „Gassenheroin“ kaufen. Oft haben Personen in Heroinprogrammen übrigens das Gefühl, dass ihnen nicht wirkliches, sondern irgendwie in seiner Wirkung beeinträchtigtes Heroin abgegeben würde. Weniger reines und mit vielen Zusatzstoffen versetztes, aber in selbstbestimmtem Rahmen konsumiertes „Gassenheroin“ ziehen sie bezüglich seiner Wirkung dem kontrolliert in den Institutionen abgegebenen Heroin vor.

\section{Zusammenfassende Schlussfolgerungen}

Wie alles menschliche Verhalten ist auch jeder Drogenkonsum mit sozialen Bedeutungen aufgeladen, die in konkreten kommunikativen Situationen produziert werden. Deshalb sollten für ein tieferes Verständnis neben pharmakologischen Wirkungen auch die Bedeutungen konkreter Konsumsituationen aufgearbeitet werden. Um Bedeutungen von Konsummustern zu rekonstruieren, müssen stets zeit- und lebensgeschichtliche Kontexte in ihrer gegenseitigen Verschränkung herangezogen werden. Tatsächlich können dem Konsum gleicher pharmakologischer Substanzen auf der zeit- ebenso wie auf der lebensgeschichtlichen Achse ganz unterschiedliche Bedeutungen zugeschrieben werden, wodurch auch ihre Attraktivität eine ganz andere ist.
Die Konsummuster des Heroins sind zunehmend vielfältiger geworden, vor allem spielen dabei Kokain und Methadon eine immer wichtigere Rolle. Reiner Heroinkonsum und länger dauernder Konsum ohne sozialmedizinische und sozialarbeiterische Betreuung sind zu einer Seltenheit geworden. Im Vergleich zur Situation von vor 30 Jahren, als praktisch jeder illegale Drogenkonsum Jugendliche und junge Leute betraf, umfasst die altersmäßige Zusammensetzung der heute Heroin Konsumierenden ein breites Spektrum. Betreuende Personen in Institutionen und Polizeikräfte sind heute oft jünger als ihre Klientinnen und Klienten.

Verfolgung und Betreuung des Heroinkonsums dürften sich in der Schweiz während der letzten drei Jahrzehnte mindestens in einem gewissen Maß scheinbar paradox auf die Attraktivität dieses Drogenkonsums ausgewirkt haben. Es war befürchtet worden, durch den Ausbau von Drogenhilfeeinrichtungen könnte der Drogenkonsum attraktiver gemacht, insbesondere die Risiken in den Augen von möglicherweise gefährdeten Personen verharmlost werden. Das Gegenteil scheint sich ereignet zu haben. So dürften etwa die Heroinprogramme unerwarteterweise in dem Sinn präventiv wirken und Außenstehende abschrecken, als sie den einst rebellischen Heroinkonsum zu einer peinlich überwachten chronischen Krankheit machen. 


\section{Literatur}

${ }^{1}$ Angst J, Baumann U, Müller U et al. Epidemiologie des Drogenkonsums im Kanton Zürich. Repräsentative Umfrage bei 6315 Zürchern und 1381 Zürcherinnen (Alter: 19 Jahre). Arch Psychiat Nervenkr 1973; 217: $11-24$

2 Battegay R, Mühlemann R, Hell D et al. Alkohol, Tabak und Drogen im Leben des jungen Mannes. Untersuchung an 4082 Schweizer Rekruten betreffend Suchtmittelkonsum im Zivilleben und während der Rekrutenschule. Basel: Karger, 1977

${ }^{3}$ Bickel P, Bösch H, Uchtenhagen A. Stoff und Applikationsart beim Drogenkonsum Jugendlicher. Sozial- und Präventivmedizin 1976; 21 : $31-37$

${ }^{4}$ Hornung R, Schmidtchen G, Scholl-Schaaf M. Drogen in Zürich. Verbreitung und Hintergründe des Drogenkonsums Jugendlicher - Ergebnisse einer Motivstudie. Bern: Hans Huber, 1983

${ }^{5}$ Ladewig D. Gesamtschweizerische Enquête über die Häufigkeit des Medikamenten- und Drogenmissbrauchs. Schweizerische Ärztezeitung 1973; 28: $971-975$

${ }^{6}$ Sieber M, Angst J. Drogen-, Alkohol- und Tabakkonsum. Ein Beitrag zur Epidemiologie und Ätiologie bei jungen Erwachsenen. Bern: Huber, 1981

7 Uchtenhagen A, Zimmer-Höfler D. Heroinabhängige und ihre „normalen“ Altersgenossen. Herkunft, Lebenssituation, Zweijahresverlauf im Quervergleich. Bern: Haupt, 1985

${ }^{8}$ Wirz G. Katamnese der ersten 50 Fixer aus dem Drop-in Zürich. Medizinische Fakultät. Zürich: Universität Zürich, 1987

${ }^{9}$ Bundesamt für Gesundheit (BAG). Prävalenzschätzungen der Heroinabhängigkeit in der Schweiz. Trend stieg bis 1993/94, dann Trendumkehr bis 1998. Bern: BAG Bulletin, 2000: 396-399

${ }^{10}$ Estermann J, Herrmann U, Hügi D et al. Sozialepidemiologie des Drogenkonsums: zu Prävalenz und Inzidenz des Heroin- und Kokaingebrauchs und dessen polizeiliche Verfolgung. Berlin: Verlag für Wissenschaft und Bildung, 1996

${ }^{11}$ Estermann J. Doch kein zunehmender Heroin- und Kokainkonsum in der Schweiz? (Letter to the editor). Sozial- und Präventivmedizin 1998; 43: 173- 174

12 Knolle H, Abelin T. Die Epidemie des Heroin- und Kokainkonsums in der Schweiz 1979-1997. In: Bundesamt für Gesundheit (BAG) (Hrsg). Suchtforschung des BAG. Band 1/4: Grundlagenforschung. Bern: BAG, 2000: $105-114$

${ }^{13}$ Maag V. Estimated trends in the prevalence of heroin addiction in Switzerland. A multiple-indicator approach. Eur Addict Res 2003; 9: $176-181$

14 Schweizerische Fachstelle für Alkohol- und andere Drogenprobleme. Zahlen und Fakten zu Alkohol und anderen Drogen, 2003 (CD-ROM). Lausanne: SFA, 2003
${ }^{15}$ Schweizerische Fachstelle für Alkoholprobleme (SFA), Université de Lausanne/DEEP-HEC. Soziale und präventive Aspekte des Drogenproblems unter besonderer Berücksichtigung der Schweiz. Lausanne, 1990

${ }^{16}$ Aarburg HP. Heroindampfscheibenwirbel: Eine kulturanthropologische und ethnopsychoanalytische Studie des Folienrauchens in Zürich zwischen 1990 und 1995. Berlin: Verlag für Wissenschaft und Bildung, 1998

${ }^{17}$ Christen L, Christen S, Grob PJ et al. Verlaufsevaluation der Zürcher Methadonabgabe-Poliklinik der ARUD „Zokl1“ 1992-1996 aufgrund von psychosozialen, konsumdefinierten und serologischen Parametern. In: BAG (Hrsg). Evaluationen des BAG im Suchtbereich 1990 - 2000. Band 4. Bern: BAG, 2002: 56-163

18 Dobler-Mikola A, Zimmer-Höfler D. KlientInnen drogentherapeutischer Gemeinschaften in der Schweiz zwischen 1978/79 und 1990/91. Drogalkohol 1993; 17: 69-82

${ }^{19}$ Stauffacher M. Behandlungsanfragen von Drogenabhängigen in der Stadt Zürich 1991 - 1993: die Nutzung von prozessproduzierten Daten zur Untersuchung von Entwicklungen im Drogenkonsumverhalten. In: BAG (Hrsg). Evaluationen des BAG im Suchtbereich 1990-2000. Band 4. Bern: BAG, 2002: $191-197$

${ }^{20}$ Tschan F. Methadonprogramme im Kanton Bern 1979-92. In: BAG (Hrsg). Evaluationen des BAG im Suchtbereich 1990-2000. Band 4. Bern: BAG, 2002: 164-169

${ }^{21}$ Zinberg NE. Drug, set, and setting. The basis for controlled intoxicant use. New Haven: Yale University Press, 1984

22 Erdheim M. Die gesellschaftliche Produktion von Unbewusstheit. Eine Einführung in den ethnopsychoanalytischen Prozess. Frankfurt: Suhrkamp, 1982

${ }^{23}$ Devereux G. Angst und Methode in den Verhaltenswissenschaften. München: Hanser, 1973

24 Strauss A, Corbin J. Grounded Theory. Grundlagen qualitativer Sozialforschung. Weinheim: Beltz, 1996

${ }^{25}$ Nigg H. Wir wollen alles, und zwar subito! Die Achtziger Jugendunruhen in der Schweiz und ihre Folgen. Zürich: Limmat Verlag, 2001

${ }^{26}$ Vogler G, Bänziger C. Nur saubergekämmt sind wir frei. In: eco-Verlag (Hrsg). Drogen und Politik in Zürich. Zürich, 1990

${ }^{27}$ Christiane F. Wir Kinder vom Bahnhof Zoo. Hamburg: Stern, 1978

${ }^{28}$ Eisner M. Städtische Drogenmärkte und Strassenraub: das Beispiel Zürich. In: Eisner M (Hrsg). Das Ende der zivilisierten Stadt? Die Auswirkungen von Modernisierung und urbaner Krise auf Gewaltdelinquenz. Frankfurt: Campus, 1997: 230-252

${ }^{29}$ Bundesamt für Gesundheit (BAG). Die Schweizer Drogenpolitik. Bern: Bundesamt für Gesundheit, 2000 